\section{Advances in research on evoked potentials}

\author{
D. Regan
}

Evoked Potentials. Edited by C. Barber. Pp. 614. (MTP Press: Lancaster, UK, 1980.) £29. Clinical Uses of Cerebral, Brainstem and Spinal Somatosensory Evoked Potentials. Edited by J.E. Desmedt. Pp. 354. (Karger: Basel, 1980.) SFr 138, \$82.75. Cognitive Components in Cerebral Event-Related Potentials and Selective Attention. Edited by J.E. Desmedt. Pp. 322. (Karger: Basel, 1979.) SFr $118, \$ 70.75$.

IT HAS been known for almost 100 years that the human brain continuously generates an electrical oscillation called the electroencephalogram (EEG) that can be recorded from electrodes held onto the scalp. The EEG can reach an amplitude of $100 \mu \mathrm{V}$, so that the brain's $0.1-10 \mu \mathrm{V}$ responses to auditory, visual, tactile or olfactory stimulation are usually hidden in the EEG. Consequently, these electrical responses to sensory stimulation, called evoked potentials, were almost unexplored until automatic signal-processing devices, developed in the $1950 \mathrm{~s}$, were used to separate evoked potentials from the brain's background activity. Part A of Barber's book is an excellent, concise survey of these signal-processing techniques and evoked potential methodology.

The three books reviewed here together cover all the main directions of current evoked potential research. Many contributors emphasize the present clinical value and future promise of evoked potential recording as an effectively riskfree means of objectively testing hearing, vision and spinal cord function. Clearly, a battery of objective sensory tests would meet a need, particularly with neonates and young infants, where the available methods for testing sensory function are imprecise. In adults, evoked potential tests can distinguish between organic and psychogenic disorders, and practical successes with adult patients are described by authors in all three books. In particular, short-latency evoked potentials recorded from auditory brainstem structures have proved their worth both in the neurological clinic and in assessing hearing disorders, and evoked potentials produced by visual patterns are widely used as an aid to diagnosing multiple sclerosis. Auditory brainstem responses are a promising means of testing infants' hearing, and a technically difficult method for recording from electrodes over the spinal cord has been successfully used with infant patients, but so far visual evoked potentials seem to have had comparatively little impact on the visual testing of infants and the management of amblyopia. Part A of Barber's

book identifies limitations in our understanding of visual evoked potential generation that must be overcome if the method is to fulfil its exciting promise in paediatric neuro-ophthalmology.

Evoked potential recording is the only widely available, risk-free means of objectively exploring the activities of normal human brains (analysis of the brain's magnetic field is still at an early stage of development). Consequently, evoked potential recording has a special appeal to scientists interested in aspects of brain activity that may be peculiar to the human species. Rather than studying evoked potentials generated by sensory stimuli, a second line of research explores scalp potentials produced or modified by thinking processes. Several authors in Desmedt's book on cognition show how evoked potentials can be used not only to study the brain activities associated with mental evaluation of incoming sensory information, but also how to study cognitive aspects of brain function including selective attention.

All three books are collections of contributions by different authors, each of whom focuses chiefly on his own research area. Although this style of book can be assembled more quickly and with less individual toil than is the case when the writing is undertaken by only one or two authors, there are disadvantages for the reader. These include greater difficulty in following a coherent thread through successive chapters, and some unevenness in the standard of different authors' contributions. Since each of the books edited by Desmedt addresses a restricted topic these drawbacks are less evident than in Barber's book. Desmedt's two books provide researchers and clinicians with summaries of current research in cognitive evoked potential studies and in somatosensory evoked potentials that are as useful as any available. However, it is a

\section{Protein synthesis from start to finish}

\section{P. J. Ford}

From DNA to Protein: The Transfer of Genetic Information. By M. Szekely. Pp. 284. (Macmillan Press: London, 1980.) Hardback £18, \$35.95; paperback £9.95.

From DNA to Protein is an up-to-date (early 1979) account of that fascinating but ill-defined area of biology comprising some genetics, some biochemistry and some macromolecular structure. The book has been written for advanced undergraduates and postgraduate students, but is also intended for research workers wishing to keep up with molecular biology.

My initial reaction to the contents pages was very favourable since the book is logically ordered, starting with a pity that substantial linking commentaries were not incorporated, since these would have made both books more effective as introductory texts. Although its inclusion may have delayed publication, the edited discussion on pattern responses in Barber's book adds considerably to the value of the text. Several more such discussions could have been added with advantage.

Barber's book is based on the 1978 evoked potential conference held in Nottingham, and resembles in some ways a book based on the 1974 Brussels conference (Visual Evoked Potentials in Man. Edited by J.E. Desmedt. Clarendon: Oxford, 1977). Some impression of progress in evoked potentials research can be obtained by comparing these two volumes. What emerges is that the relation between cognition and long-latency evoked potentials is more complex than was thought to be the case in 1974; it now seems that several late waves exist, differing from one another in their brain origins and psychological correlates. Our basic understanding of visual evoked potentials advanced substantially before 1974 , but progress seems to have slowed since then. The few researchers attempting to develop evoked potentials as muchneeded objective measures in psychiatry have continued to encounter formidable problems including the broadness of clinical diagnostic classifications. In contrast, the use of visual evoked potentials in the neurology clinic, a research technique in 1974, is now routine in a large number of hospitals. Perhaps the major new thrusts in basic research and in clinical applications have been in auditory brainstem and somatosensory evoked potentials, and these topics are well covered both by Barber and by Desmedt. $\square$

D. Regan is I.W. Killam Research Professor in the Department of Physiology and Professor of Ophthalmology at Dalhousie University, Halifax, Nova Scotia.

description of DNA structure and replication, followed by transcription and messenger RNA structure and leading into a discussion of translation. There is also a chapter on methods of nucleotide sequence determination which, for some undergraduate teaching purposes, might be too specialized. Such detailed attention nevertheless does indicate the overwhelming role of technology in forcing advances in this area, a point which I think cannot be made too often.

Reading the book in more detail I found that descriptions of particular systems were generally easily followed although there are many places where more careful editing would have improved communication. Some more obvious errors might also have been removed such as the "thousands (of ribosomal RNA genes) in Drosophila"' (p. 16) or more seriously the suggestions that satellite DNAs are small molecules (p. 28) and that yeast has a single 